\title{
Free Radical Polymers with Tunable and Selective Bio- and Chemical Degradability
}

\author{
Jos M.J. Paulusse ${ }^{1}$, Roey J. Amir ${ }^{1}$, Richard A. Evans ${ }^{2}$, and Craig J. Hawker ${ }^{1,}{ }^{*}$ \\ ${ }^{1}$ Departments of Chemistry and Biochemistry, Materials Department, Materials Research \\ Laboratory, University of California, Santa Barbara, California 93106-9510, USA \\ ${ }^{2}$ CSIRO Molecular \& Health Technologies, Bag 10, Clayton VIC, 3169, Australia
}

\section{Abstract}

A versatile synthetic strategy has been developed which enables the facile incorporation of cleavable functional groups, i.e. esters, thioesters and disulfides, into the carbon-carbon backbone of vinylbased polymers. Through the synthesis of novel cyclic monomers, RAFT-mediated radical ringopening copolymerizations with traditional vinyl monomers such as methyl methacrylate, $\mathrm{N}, \mathrm{N}-$ dimethylaminoethyl methacrylate and 2-hydroxyethyl methacrylate leads to the introduction of controlled degradability into these widely used vinyl copolymer systems. An additional benefit of this strategy is the inherent versatility available through the incorporation of cyclic monomers containing diverse functional groups such as esters, thioesters, disulfides and silyl ether units that allow degradation under basic/acidic, reductive or enzymatic conditions. By integrating multiple, orthogonal cyclic monomers into linear copolymer backbones, well-defined systems with programmable degradation profiles are obtained which allows for tunable, selective and step-wise degradation of the vinyl polymer backbones.

\section{Keywords}

Radical polymerization; macrocycles; RAFT-polymerization; ring opening polymerization; biodegradability

\section{Introduction}

\begin{abstract}
As vinyl-based polymers are used in a wider and wider variety of applications, especially in the area of biomaterials, the degradability of these systems becomes a critical issue. However, in contrast to many other polymer families, such as polyesters derived from ring-opening polymerization, the carbon-carbon backbone of vinyl-based polymers does not readily undergo cleavage or degradation reactions. As a result, degradability of these systems does not occur under normal conditions and the polymer backbone is persistent, especially in biological environments. This is unfortunate since the high tolerance of controlled/living free radical polymerization ${ }^{1-5}$ towards different monomers and functional groups, coupled with facile synthetic techniques, has led to a wealth of new materials and applications for vinyl-based polymers. ${ }^{6}$ To address this challenge, there is a fundamental need for the development of a
\end{abstract}

Hawker@mrl.ucsb.edu; Fax: +1 805893 8797; Tel: +1 8058937161.

Support Information Available

The synthesis and characterization data for monomers, intermediates and polymers is available free of charge via the Internet at http://pubs.acs.org. 
versatile and controlled method for the incorporation of reactive and potentially orthogonal functional groups into the backbone of vinyl based polymers.

Several distinct approaches have been used to introduce degradable functionalities into polymeric structures. One of the most practical approaches is the use of a bifunctional, degradable monomer, ${ }^{7-10}$ which acts as a crosslinker, leading primarily to network structures. More recently, the preparation of well-defined star polymers using bifunctional monomers has been demonstrated by Matyjaszewski and coworkers, ${ }^{11}$ though linear polymers cannot be obtained with this strategy. An approach that does allow for the preparation of linear materials is the condensation of smaller, oligomeric precursors. These pre-polymers may be prepared from a bifunctional initiator containing the degradable unit, ${ }^{12}$ followed by coupling using an efficient reaction such as atom transfer radical coupling (ATRC).$^{13}$ Alternatively, a regular bifunctional initiator can be used and the degradable unit incorporated during a subsequent condensation step. For example, reversible addition fragmentation chain transfer (RAFT) polymerization typically results in a dithioester chain end. ${ }^{14}$ This group may be aminolysed to give the corresponding thiol, ${ }^{15}$ subsequent oxidation of this thiol then leads to linear polymers containing disulfide linkages in the backbone.${ }^{16}$ Linearity of the final polymeric structures is ensured, however the second condensation step results in a significant increase in polydispersity. Moreover, these techniques require several synthetic steps, which also limits their applicability.

Given these challenges, one of the most promising strategies for incorporating backbone functional groups is by radical ring opening polymerization of cyclic monomers. ${ }^{17}$ Several very different classes of cyclic monomers have been developed. Bailey and coworkers were the first to prepare polyesters via radical ring-opening polymerization of 5,6-benzo-2methylene-1,3-dioxepane (BMDO). ${ }^{18}$ The copolymerization of this cyclic monomer with $\mathrm{n}$ butyl acrylate was recently investigated in detail by Huang et al. and a preliminary degradability study of the resulting copolymers was carried out. ${ }^{19}$ In expanding this field, Rizzardo and Evans have developed a series of cyclic monomers based on allylic sulfides. ${ }^{20-22}$ In this system, radical attack on the alkene is followed by radical transfer to the sulfur atom resulting in opening of the ring, formation of a thiyl-radical and addition of subsequent monomers. Although primarily investigated for their low-shrinkage properties, which are characteristic of cyclic monomers, the ability to employ main-chain functionality via radical polymerization also received attention. Chaumont investigated copolymerizations of styrene with cyclic allylic sulfides and the hydrolytic degradation of these copolymers. ${ }^{23}$ However, a significant discrepancy was found between the number of cyclic monomers incorporated and the actual amount of hydrolyzed groups, most likely caused by unwanted crosslinking during the polymerization. $^{24}$

We report here the synthesis of a library of novel cyclic monomers, containing ester, thioester and disulfide functionalities that can be used for the preparation of well-defined linear copolymers via RAFT polymerization with methyl methacrylate (MMA), $N, N$ dimethylaminoethyl methacrylate (DMAEMA) and 2-hydroxyethyl methacrylate (HEMA). A critical feature of these materials is the ability to incorporate one or more orthogonally reactive units into the vinyl backbone leading to tunable, selective and step-wise degradation while at the same time introducing a site for backbone functionalization (Figure 1).

\section{Results and Discussion \\ Monomer synthesis}

The parent cyclic allylic sulphide monomer 2, containing an 11-membered ring and a single ester unit, was prepared following the procedure developed by Evans, Rizzardo and coworkers. $20,24,25$ Nucleophilic substitution of $\alpha$-bromomethyl acrylic acid with 6-mercapto-1-hexanol 
yielded the hydroxyl acid $\mathbf{1}$ as a white crystalline solid (92\%), which was then cyclized under high dilution conditions in the presence of Mukaiyama's reagent. ${ }^{26}$ (Scheme 1) This afforded the cyclic monomer $\mathbf{2}$ as a white crystalline solid in $30 \%$ yield with the major byproducts being identified as dimers, trimers, etc. as well as adducts of $\mathbf{1}$ with the cyclization agent.

The limited versatility of the hydroxyl acid $\mathbf{1}$ prompted the development of a more general synthetic intermediate which would allow a much greater range of cyclic monomers to be prepared. To this end, the $\alpha$-bromomethyl acrylic acid was reacted with 3-mercaptopropionic acid to yield the stable dicarboxylic acid $\mathbf{3}$ in excellent yield (Scheme 2). The presence of two carboxylic acid groups in $\mathbf{3}$ then allows a wide range of cyclic derivative to be prepared by esterification using readily available diols. Activation of $\mathbf{3}$ by conversion to the corresponding bis-acid chloride was accomplished by reaction with oxalyl chloride, using $N, N$ dimethylformamide (DMF) as a catalyst. While the bis-acid chloride proved to be highly susceptible to hydrolysis, it could be effectively used after thorough removal of excess oxalyl chloride under vacuum and stored for short periods of time. To demonstrate the versatility of this synthetic approach, the bis-acid chloride was reacted with 1,5-pentanediol to give the diester 5 ( $25 \%$ yield), while reaction with 1,6-hexanedithiol gives the bis-thioester cyclic monomer $\mathbf{6}$ and 2,2'-dithiodiethanol afford the disulfide cyclic monomer $\mathbf{4}$ as a white crystalline material. For monomer $\mathbf{4}$, crystals suitable for $\mathrm{x}$-ray crystallography were obtained via slow solvent evaporation from a mixture of dichloromethane and $n$-hexane (50:50 by volume). The molecular structure of $\mathbf{4}$ is depicted in Figure 2 and clearly shows the presence of a 15-membered ring incorporating 2 ester groups and a disulphide linkage.

An alternative strategy for the synthesis of the cyclic monomers involves reaction of the acid chloride of $\mathbf{3}$ with an excess of ethylene glycol, giving rise to the chain-extended diol $\mathbf{7}$ which could be cyclized through reaction with dimethyldichlorosilane (Scheme 3). In this case, purification of the crude reaction mixture by column chromatography yields the desired silylether monomer $\mathbf{8}$ which contains a 16 membered ring and orthogonally reactive ester and silyl ether units. For all the cyclic monomers described above, ${ }^{1} \mathrm{H}$ and ${ }^{13} \mathrm{C}$ NMR, ESI-MS and FT-IR confirmed the formation of the desired structures and size exclusion chromatography (SEC) was used to confirm the absence of higher oligomers. All cyclic monomers were stable for several months at $-20^{\circ} \mathrm{C}$ showing no signs of coloration or decomposition.

\section{Copolymerizations}

In order to understand the copolymerization behavior of these cyclic monomers and the effect, if any, of changing the ring size, conventional free radical polymerization using AIBN of the parent cyclic monomer $2(1 \mathrm{~mol} \%)$ with MMA was initially studied. In accord with previous studies, ${ }^{21}$ high molecular weight, polydisperse polymer was obtained (number average molecular weight, $\mathrm{M}_{\mathrm{n}} \sim 2.4 \times 10^{4} \mathrm{~g} / \mathrm{mol}$; polydispersity, PDI $\sim 2.0$ ), with co-monomer incorporation corresponding to the feed ratio (1\%). However the intrinsically high polydispersity and poorly controlled nature of the copolymerization, prompted an examination of RAFT polymerization, using methoxycarbonylbenzyl dithiobenzoate ${ }^{27}$ as RAFT-agent, in order to achieve control over molecular weight as well as polydispersity.

Copolymers P1-4 based on MMA and varying feed ratios of cyclic monomers 2, 4-7 were prepared under standard RAFT conditions (Figure 3). ${ }^{1} \mathrm{H}$ NMR spectroscopy on the crude reaction mixtures confirmed approximately equal rates of consumption for MMA and the cyclic monomers at approximately $50 \%$ conversion with unique resonances for repeat units derived from the cyclic monomers (Figure 4). The copolymers were purified by precipitation in hexanes, and initially analyzed by SEC. At low incorporation ratios (1\%), polydispersity indices (PDI) of 1.2-1.3 were obtained for all copolymers with the PDI increasing to 1.4-1.5 as the cyclic monomer content increased to $5 \%$. At high feed ratios (ca. 10+\%), the polydispersity increased to over 2.0 due to radical addition of growing chains to the 1,1 - 
disubstituted alkene units along the backbone. For feed ratios of less than $5 \mathrm{~mol} \%$, the number average molecular weights $\left(\mathrm{M}_{\mathrm{n}}\right)$ of the copolymers were in close agreement with the theoretical molecular weights $\left(3 \times 10^{4} \mathrm{~g} / \mathrm{mol}\right)$ in all cases except for the silyl ether based cyclic monomer 7. For a $1.6 \mathrm{~mol} \%$ feed ratio of 7 , the experimental $\mathrm{M}_{\mathrm{n}}$ of $1.5 \times 10^{4} \mathrm{~g} / \mathrm{mol}$ was significantly decreased when compared to the theoretical MW of $3 \times 10^{4} \mathrm{~g} / \mathrm{mol}$. A possible reason for this discrepancy is the facile degradation of the silyl ether units, ${ }^{28}$ which results in chain scission at the silyl ether linkages during purification and characterization. Additional studies showed that increased handling and storage leads to further decreases in molecular weight of the copolymers based on $\mathbf{7}$ with the final molecular weight corresponding to that expected for full cleavage of all silyl ether units in the backbone.

Having demonstrated the feasibility of incorporating functionalized cyclic monomers and their copolymerization behavior with MMA, the ability to form copolymers with other commodity monomers was investigated. While MMA is a good model for hydrophobic materials, one of the primary uses for these functionalized cyclic monomers is for controlling the degradation of materials for biological and medical applications. To investigate these systems, a range of copolymers were prepared, based on water-compatible monomers such as DMAEMA and HEMA. Initial copolymerizations were carried out with 1 mol \% of cyclic monomers 2, 4, 5 and $\mathbf{6}$ and either DMAEMA, which leads to the fully water-soluble copolymers P6-9, or HEMA, resulting in water-swellable copolymers P10-14. The DMAEMA copolymers were purified by dissolution in dichloromethane and precipitation in cold hexanes, yielding viscous oils. In contrast, the HEMA copolymers were dissolved in methanol and precipitated in diethyl ether, yielding the copolymers as pink powders. The final copolymers were fully characterized using a combination of NMR, IR spectroscopy as well as SEC. For both series of polymers, the molecular weights and polydispersities were in good agreement with the targeted molecular weights/polydispersities and the level of cyclic monomer incorporation $v s$. monomer feed ratio consistent with the observations for the MMA-based copolymers. The ease of changing the vinyl monomer in these systems suggests that the incorporation of these functionalized cyclic monomers is a general strategy and can be applied to a wide variety of polymeric backbones and applications. Significantly it was also shown that the 1,1-disubstituted alkene units of the polymer backbone which results from ring opening of the cyclic monomer is an additional site for orthogonal chemical modification of these materials. Thiol-ene or Michael additions across the double bond were shown to be possible without any observed degradation of the polymer backbone. These results show that in-chain double bond is also a point of chemistry that can be exploited for mild modification of the polymer although the efficiency of the reaction was reduced due to the steric environment of the double bond.

\section{Chemical degradation}

The ability to prepare a range of degradable copolymers under controlled conditions allows the cleavage of these systems to be studied in detail. While the level of cyclic monomer incorporation could be determined by ${ }^{1} \mathrm{H}$ NMR, the random nature of incorporation and the presence of functional linkers in the backbone was best demonstrated through chemical degradation. As all of the cyclic monomers contain either esters or thioesters groups, chemical degradation with sodium methoxide was initially chosen as the basic screen for comparing the different vinyl backbones at a variety of different feed ratios. For the MMA and DMAEMAbased copolymers, reaction with sodium methoxide in a mixture of THF/methanol was monitored by the change in molecular weight over time by SEC. Significantly, it was shown that the ester and thioester linkages in the backbone were completely cleaved after only 30 minutes at room temperature with the molecular weight of the final linear polymer correlating closely with the expected molecular weight based on the feed ratio of the cyclic monomer. Figure 4 shows a series of SEC traces for MMA-based copolymers P1a-d, derived from the 11-membered, monoester cyclic monomer 2, and copolymers P2a-c, based on the 15- 
membered, diester/disulfide cyclic monomer $\mathbf{4}$, at different incorporation ratios (solid lines), and their corresponding degradation products (dashed lines). In calculating the molecular weights for the polymers after degradation, the degree of polymerization for the starting copolymer was multiplied by the molar incorporation of the cyclic monomer with the assumption that every repeat unit derived from a cyclic monomer will undergo degradation (i.e. for a 30,000 amu MMA-based copolymer with $5 \mathrm{~mol} \%$ incorporation of cyclic repeat units, DP of the degraded copolymer is approximately $300 \times 0.05=15$, giving an expected molecular weight of 1500). For each sample, the correlation between calculated and experimental molecular weight for the degradation products was high with the polydispersity increasing only marginally for the degradation products.

In the case of HEMA-based copolymers, the presence of the hydroxyl groups did not allow the sodium methoxide strategy described above to be successfully employed. Transesterification under acidic conditions $\left(\mathrm{MeOH} / \mathrm{H}_{2} \mathrm{SO}_{4}\right)$ was therefore applied to the HEMA-based copolymers which resulted in rapid degradation and the observed decrease in molecular weight was again in good agreement with that expected from the level of cyclic monomer incorporation. It should also be noted that control experiments in which the same degradation experiments were performed on the corresponding homopolymers of MMA, DMAEMA and HEMA did not result in any detectable changes in molecular weight or polydispersity. This clearly shows that the degradation chemistry is not causing decomposition of the vinyl polymer backbone or side chains in the absence of repeat units derived from the cyclic monomers.

\section{Water-soluble PHEMA}

To fully demonstrate the potential for controlled degradation of vinyl backbone copolymers in biomaterial applications, a thorough investigation into synthesis and degradation of PHEMAbased copolymers was undertaken. The motivation behind this study was the wide use of PHEMA in many biological applications coupled with the unusual solubility profile for the homopolymer. Recent investigations ${ }^{32}$ into the water-compatibility of PHEMA reveal that the polymer is only fully water soluble when the molecular weight is low and the degree of polymerization (DP) below 20. Limited water solubility is observed for DP's up to 45 while for high molecular weight materials, $M_{n}>10 \mathrm{~kg} / \mathrm{mol}$, linear polymers are insoluble and only swell in water or buffer solutions. This creates the conundrum of desiring high molecular weight material for applications due to superior mechanical and physical properties while requiring low molecular weight material to ensure complete clearance from the body. In order to develop a PHEMA copolymer that has fully water-soluble degradation products, an incorporation ratio of 5-10\% of cleavable functionalities into the PHEMA backbone was targeted. PHEMA-based copolymers P14 containing 5-10\% of cyclic disulfide monomer 4 were therefore prepared with ${ }^{1} \mathrm{H}$ NMR analysis confirming the level of incorporation and SEC revealing a molecular weight of $7.4 \times 10^{4} \mathrm{~g} / \mathrm{mol}$ and PDI of 1.86 for the $5 \mathrm{~mol} \%$ sample. As predicted, this material proved to be swellable, though insoluble, in water. Significantly, on addition of a hydrazine solution ( $30 \mathrm{wt} \%$ in water), a dramatic increase in swelling and subsequent dissolution of the PHEMA was observed within 20 minutes at room temperature. The final degradation products were isolated by drying and shown to have a degree of polymerization of ca. 21, close to the theoretical value of 22. As expected the oligomeric PHEMA units with this low DP could be repeatedly solubilized in water.

\section{Orthogonal degradation}

The ability to design and copolymerize cyclic monomers incorporating different reactive functional groups raises the interesting possibility of multiple and orthogonal degradation processes being designed into the one material. Two strategies are possible, incorporation of different functional groups into a single cyclic monomer and incorporation of multiple cyclic monomers containing different functional groups into the same polymeric backbone. To 
demonstrate the first strategy, the cyclic monomer 6 was designed to contain both thioester as well as ester groups. In this case, degradation with sodium thiomethoxide is chemoselective for the thioester group and does not degrade regular esters. ${ }^{29}$ Copolymers P4, P9 and P13 containing varying amounts of the thioester cyclic monomer $\mathbf{6}$ were therefore degraded with sodium thiomethoxide and again degradation was observed to be complete after $30 \mathrm{~min}$. Under identical conditions, the corresponding MMA-based copolymers of cyclic monomer 2 containing only regular esters did not degrade even when the degradation temperature was raised to $70^{\circ} \mathrm{C}$ for prolonged periods. This demonstrates that the thioester containing monomers can be used to impart orthogonal degradation in the presence of ester groups.

As an example of orthogonal degradation using a combination of different cyclic monomers, a random terpolymer P15 was prepared from MMA, 0.3\% of cyclic disulfide monomer $\mathbf{4}$ and $0.8 \%$ of cyclic diester monomer $\mathbf{5}$. The choice of the disulfide monomer $\mathbf{4}$ was governed by the potential for facile chemical degradation under conditions that do not degrade esters. As a demonstration, a random copolymer of $\mathbf{4}$ and MMA, P2a, was dissolved in THF and either hydrazine ${ }^{30}$ or tributylphosphine ${ }^{31}$ added. After less than 15 minutes at room temperature, degradation of the disulfides was complete under both conditions (Scheme 4). In contrast, degradation of MMA copolymers containing only ester functionalities did not occur under these conditions. For the terpolymer P15 $\left(\mathrm{M}_{\mathrm{n}}=9.1 \times 10^{4} \mathrm{~g} / \mathrm{mol}\right.$, PDI $\left.=1.28\right)$, the starting polymer was first treated with hydrazine which resulted in a reduction in the molecular weight to $4.2 \times 10^{4} \mathrm{~g} / \mathrm{mol}$. Subsequently, the primary degradation product obtained from the hydrazine reaction was secondarily degraded with sodium methoxide, resulting in a further reduction of molecular weight to $1.2 \times 10^{4} \mathrm{~g} / \mathrm{mol}$. This step-wise degradation agrees well with the estimated block sizes based on the relative incorporation of 4 and $5\left(3.6 \times 10^{4} \mathrm{~g} / \mathrm{mol}\right.$ and $1.0 \times 10^{4} \mathrm{~g} / \mathrm{mol}$ respectively) and shows the potential to tune degradation profiles through the use of two or more cyclic monomers with different functional groups (Figure 5).

\section{Enzymatic degradation}

The ability to control and optimize the chemical degradation of vinyl polymer backbones then raises the interesting possibility of enzymatically cleaving the backbone under physiological conditions. To examine this feature, the fully water soluble DMAEMA-based copolymers P7 derived from the 15-membered cyclic monomer containing 2 ester groups were prepared and then subjected to degradation in the presence of commercially available pig liver esterase (PLE). The copolymers were dissolved in water, PLE was added and the reaction mixture stirred for 4 hours at $37^{\circ} \mathrm{C}$. After isolating the degradation products, SEC analysis showed the generation of lower molecular weight products fully consistent with complete hydrolysis of the backbone ester groups (Figure 7). Analysis of DMAEMA-based copolymers prepared from other cyclic monomers also showed efficient enzymatic degradation to give products having molecular weights in agreement with theory. These results demonstrate that a variety of activation strategies (i.e. chemical or enzymatic) can be used to degrade the backbone of radically produced vinyl copolymers.

\section{Conclusions}

The challenge of introducing a variety of degradable units into the backbone of vinyl-based copolymers has been addressed through the synthesis of a family of cyclic allylic sulphide monomers that incorporate different functional groups, e.g. esters, thioesters and disulfides, into cyclic allylic sulfides. These cyclic monomers were readily copolymerized with commercially important and widely studied vinyl monomers such as MMA, DMAEMA and HEMA under RAFT conditions leading to give good control over molecular weight and polydispersity. Significantly, the combination of different functional groups within the same monomer, or the use of different cyclic monomers within the same copolymer, allows the 
introduction of different functional groups into the polymer backbone and permits step-wise, orthogonal cleavage. This additional level of control over polymer degradation enables a range of novel applications for vinyl polymers in biological systems. Expansion of this synthetic methodology to include a wider range of polymer backbone functionalities is currently underway, as are more detailed studies of the polymer degradation process.

\section{Experimental}

\section{General procedures}

${ }^{1} \mathrm{H}$ NMR (400 MHz) and ${ }^{13} \mathrm{C}$ NMR (100 MHz) spectra were recorded on a Varian 400 spectrometer in deuterated chloroform unless stated otherwise. Chemical shifts are reported in $\mathrm{ppm}$ and referenced to tetramethylsilane and chloroform $\left({ }^{1} \mathrm{H}\right.$ and $\left.{ }^{13} \mathrm{C}\right)$. Column chromatography was performed on a Biotage SP1 Flash Purification System using FLASH 40 $+\mathrm{M}$ and FLASH $25+\mathrm{M}$ cartridges. Size exclusion chromatography was performed on a Waters 2695 Separation Module equipped with four $5 \mu \mathrm{m}$ Water columns $(300 \times 7.7 \mathrm{~mm})$, connected in series with increasing pore size $\left(10^{2}, 10^{3}, 10^{4}\right.$, and $\left.10^{6} \AA\right)$, and a Waters 2414 Refractive Index Detector and Waters 2996 Photodiode Array Detector. For size exclusion chromatography analysis (SEC) of poly(methyl methacrylate) polymers THF was used as eluent, while for the analysis of poly $(N, N$-dimethylaminoethyl methacrylate) and poly(2hydroxyethyl methacrylate) polymers DMF was used as eluent. In all cases, poly(methyl methacrylate) standards were used for calibration. Infrared spectra were recorded on a Perkin Elmer Spectrum 100 with a Universal ATR sampling accessory. 6-Mercapto-1-hexanol (97\%) was purchased from Fluka, methyl methacrylate (99\%), 2-hydroxyethyl methacrylate (99+\%), $N, N$-dimethylaminoethyl methacrylate (99\%), $\alpha$-bromomethyl acryclic acid (98\%), chlorobenzene (99\%), tri-n-butylphosphine (\%), hydrazine $35 \mathrm{wt} \%$ in water and 2,2'-azobis (2-methylpropionitrile) (AIBN) (98\%) were purchased from Aldrich and 2-chloro-1methylpyridinium iodide (97\%) and sodium methoxide $30 \mathrm{wt} \%$ in methanol were purchased from ACROS. Methyl methacrylate was filtered over activated neutral alumina prior to use. Methoxycarbonylbenzyl dithiobenzoate (MCBDB) was synthesized following a literature procedure ${ }^{1}$ modified literature procedures were used to synthesize [(6-hydroxyhexyl)thiomethyl]acryclic acid and 3-methylidene-1-oxa-5-thiacycloundecan-2-one. ${ }^{2-5}$

\section{Synthesis of [(6-hydroxyhexyl)thiomethyl]acryclic acid, (1)}

Triethylamine $(5.18 \mathrm{~mL}, 37.3 \mathrm{mmol})$ was added dropwise to a cooled $\left(0^{\circ} \mathrm{C}\right)$ solution of abromomethylacrylic acid $(3.07 \mathrm{~g}, 18.6 \mathrm{mmol})$ in dichloromethane $(100 \mathrm{~mL})$. 6-Mercapto-1hexanol $(2.50 \mathrm{~g}, 18.6 \mathrm{mmol})$ was then added over a period of $15 \mathrm{~min}$, and the reaction mixture stirred for $20 \mathrm{~h}$. A solution of ammonium sulfate $(9.0 \mathrm{~g}, 78.8 \mathrm{mmol})$ and sulfuric acid $(5 \mathrm{~mL}$, $2 \mathrm{M}, 10 \mathrm{mmol})$ in water $(100 \mathrm{~mL})$ was cooled to $0^{\circ} \mathrm{C}$, and the reaction mixture poured into it. This mixture was extracted with diethyl ether $(3 \times 60 \mathrm{~mL})$, the organic phase dried with magnesium sulfate and solvent removed under reduced pressure, yielding a white solid. (3.72 $\mathrm{g}, 92 \%) \delta_{\mathrm{H}}(400 \mathrm{MHz}): 6.33(\mathrm{~s}, 1 \mathrm{H}), 5.76(\mathrm{~s}, 1 \mathrm{H}), 3.62(\mathrm{t}, 2 \mathrm{H}), 3.38(\mathrm{~s}, 2 \mathrm{H}), 2.42(\mathrm{t}, 2 \mathrm{H}), 1.58$ $(\mathrm{m}, 4 \mathrm{H}), 1.38(\mathrm{~m}, 4 \mathrm{H}) . \delta_{\mathrm{C}}(100 \mathrm{MHz}): 170.9,161.4,136.9,128.0,70.7,62.9,32.5,31.7,29.2$, 28.6, 25.4. ESI-MS: $219.10\left(\mathrm{M}+\mathrm{H}^{+}\right), 241.08\left(\mathrm{M}+\mathrm{Na}^{+}\right), 257.06\left(\mathrm{M}+\mathrm{K}^{+}\right)$FT-IR, v: 3106, 2925, 2855, 1707, 1676, 1621, 1466, 1444, 1408, 1336, 1312, 1233, 1213, 1073, 1054, 970, 896, $811,758,720$.

\section{Synthesis of 3-methylidene-1-oxa-5-thiacycloundecan-2-one, (2)}

[(6-hydroxyhexyl)thiomethyl] acryclic acid $(1.50 \mathrm{~g}, 6.87 \mathrm{mmol})$ was dissolved in a mixture of dichloromethane $(60 \mathrm{~mL})$ and triethylamine $(7.65 \mathrm{~mL}, 55.0 \mathrm{mmol})$, and added via syringe pump over a period of $8 \mathrm{~h}$ to a refluxing solution of 2-chloro-1-methylpyridinium chloride (7.02 g, $27.5 \mathrm{mmol})$ in dichloromethane $(650 \mathrm{~mL})$. The reaction mixture was cooled down to room temperature, filtered, the solvent removed under reduced pressure and the solids redissolved 
in water $(100 \mathrm{~mL})$ and extracted with diethyl ether $(3 \times 30 \mathrm{~mL})$. The extracts were dried with magnesium sulfate and removal of solvent gave an orange liquid. The liquid was purified via column chromatography, yielding a white solid. (410 mg, 30\%) Rf 0.70 (dichloromethane). $\delta_{\mathrm{h}}(400 \mathrm{MHz}): 6.11(\mathrm{~s}, 1 \mathrm{H}), 5.27(\mathrm{~s}, 1 \mathrm{H}), 4.16(\mathrm{t}, 2 \mathrm{H}), 3.35(\mathrm{~s}, 2 \mathrm{H}), 2.54(\mathrm{t}, 2 \mathrm{H}), 1.84-1.57(\mathrm{~m}$, $8 \mathrm{H}) . \delta_{\mathrm{C}}(100 \mathrm{MHz}): 166.4,138.6,126.8,66.3,32.8,30.5,25.8,24.2,23.8$, 22.8. ESI-MS: $201.09\left(\mathrm{M}+\mathrm{H}^{+}\right), 223.07\left(\mathrm{M}+\mathrm{Na}^{+}\right), 239.06\left(\mathrm{M}+\mathrm{K}^{+}\right)$. FT-IR, v: 3105, 2931, 2854, 1711, 1634, $1468,1459,1443,1390,1354,1302,1284,1236,1197,1132,1059,988,971,863,815,760$, 731.

\section{Synthesis of copolymers}

For each type of copolymer (methyl methacrylate, 2-hydroxyethyl methacrylate and $\mathrm{N}, \mathrm{N}$ dimethylaminoethyl methacrylate) a representative experimental procedure is given.

\section{Synthesis of poly(methyl methacrylate)-co-(3-methylidene-1-oxa-5-thiacycloundecan-2-one) $1 \%$, (P1a)}

A $5 \mathrm{~mL}$ ampoule with stir-bar was filled with methyl methacrylate $(0.50 \mathrm{~g}, 5.00 \mathrm{mmol})$, cyclic monomer $2(10.0 \mathrm{mg}, 50 \mu \mathrm{mol})$, chlorobenzene $(0.50 \mathrm{~g}, 4.44 \mathrm{mmol})$, AIBN (stock solution in chlorobenzene: $10 \mathrm{~g} / \mathrm{L}, 0.27 \mathrm{mg}, 1.6 \mu \mathrm{mol}$ ) and methoxycarbonylbenzyl dithiobenzoate (stock solution in chlorobenzene, $100 \mathrm{~g} / \mathrm{L}, 2.5 \mathrm{mg}, 8.3 \mu \mathrm{mol}$ ). Oxygen was removed via three freezepump-thaw cycles, and the ampoule was flame-sealed and heated at $70^{\circ} \mathrm{C}$ for $17 \mathrm{~h}$. The ampoule was opened and the reaction mixture was diluted with dichloromethane $(2 \mathrm{~mL})$ and precipitated in hexanes $(100 \mathrm{~mL})$, yielding the polymer as a pink powder. (Yield: $239 \mathrm{mg} ;{ }^{1} \mathrm{H}$ NMR: $59 \%$ conv.; GPC: $\mathrm{M}_{\mathrm{n}}=3.7 \times 10^{4} \mathrm{~g} / \mathrm{mol}, \mathrm{M}_{\mathrm{w}}=5.3 \times 10^{4} \mathrm{~g} / \mathrm{mol}$, PDI: 1.42) $\delta_{\mathrm{H}}(400 \mathrm{MHz}): 6.17(\mathrm{~s}$, $1 \mathrm{H}), 5.46(\mathrm{~s}, 1 \mathrm{H}), 4.07(\mathrm{t}, 2 \mathrm{H}), 3.60(\mathrm{~b}, \mathrm{n} \times 3 \mathrm{H}), 3.40(\mathrm{~b}, 2 \mathrm{H}), 2.43(\mathrm{~b}, 2 \mathrm{H}), 2.03-0.75(\mathrm{~b}, \mathrm{n} \times$ $5 \mathrm{H})$.

\section{Chemical degradation experiments}

Ester cleavage-Poly(methyl methacrylate) copolymers P1-4 (30 mg) were dissolved in THF $(2 \mathrm{~mL})$ and sodium methoxide ( $30 \mu \mathrm{L}$ of a $30 \mathrm{wt} \%$ solution in methanol) was added to the solution, and the mixture was stirred for $30 \mathrm{~min}$. THF was evaporated and $1.2 \mathrm{M}$ aqueous hydrochloric acid $(1 \mathrm{~mL})$ was added, and the mixture was extracted with ethyl acetate $(2 \mathrm{~mL})$. The organic phase was dried with magnesium sulfate and dried in vacuo. The polymer sample was analyzed with size exclusion chromatography. The same conditions were used for poly ( $N, N$-dimethylaminoethyl methacrylate) copolymers P6-9, but instead of aqueous hydrochloric acid, saturated aqueous sodium carbonate was used, in order to prevent protonation of the amines. Poly(2-hydroxyethyl methacrylate) copolymers P10-13 were dissolved in methanol $(2 \mathrm{~mL})$ and degraded with three drops of concentrated sulfuric acid. After $30 \mathrm{~min}$, the sample was dried and water was added to the solids. Filtration yielded the polymers, which were analyzed with size exclusion chromatography.

Disulfide reduction-Copolymers P2, P7, P11 and P14 containing disulfide moieties were dissolved in THF or methanol and hydrazine ( $30 \mu \mathrm{L}$ of a $35 \mathrm{wt} \%$ solution in water) was added to the solutions. The mixtures were stirred for $30 \mathrm{~min}$ and the same workup was employed as described before. Alternatively, $30 \mu \mathrm{L}$ of tri- $n$-butylphosphine was added to the copolymers, together with $100 \mu \mathrm{L}$ of water; the mixtures were stirred for $30 \mathrm{~min}$, followed by the same workup.

Thioester cleavage-Cleavage of thioester moieties was performed either with sodium methoxide ( $30 \mu \mathrm{L}$ of a $30 \mathrm{wt} \%$ solution in methanol) and $30 \mathrm{mg}$ of the corresponding thioester containing copolymers $(\mathbf{P 4}, \mathbf{P 9}, \mathbf{P 1 3})$, or with sodium thiomethoxide $(30 \mathrm{mg})$ and the corresponding copolymers. The same workup was employed as described above. 
Degradation of reference polymers-Homopolymers of MMA, DMAEMA and HEMA were subjected to the same degradation procedures, to ensure that no backbone linkages were broken in the degradation processes, and that no reaction with pendant ester groups on the polymer, i.e. methyl ester, 2-hydroxyethyl ester and $N, N$-dimethylaminoethyl ester, occurred.

Enzymatic Degradation-Poly(N,N-dimethylaminoethyl methacrylate) copolymer P7 (50 $\mathrm{mg})$ and $\mathbf{P 9}(50 \mathrm{mg})$ were each dissolved in water $(5 \mathrm{ml})$, pig liver esterase (PLE) $(100 \mu \mathrm{L}$ of $16.5 \mathrm{mg} / \mathrm{ml}$ solution) was added and the mixture was stirred for 4 hours at $37^{\circ} \mathrm{C}$. The $\mathrm{pH}$ was raised to 12 and the degradation products were isolated by extraction with dichloromethane. The organic phase was dried and analyzed by size exclusion chromatography.

\section{Supplementary Material}

Refer to Web version on PubMed Central for supplementary material.

\section{Acknowledgements}

J.P. acknowledges the Netherlands Organization for Scientific Research (NWO) for a Rubicon fellowship. This material is based upon work supported by the National Heart Lung and Blood Institute of the National Institutes of Health, as a Program of Excellence in Nanotechnology (HL080729) and the characterization of the polymeric materials was enabled by the use of facilities supported by the MRSEC Program of the National Science Foundation (DMR-0520415). Dr. Guang Wu is kindly acknowledged for crystal structure determination.

\section{References}

1. Ma J, Cheng C, Wooley KL. Macromolecules 2009;42:1565-1573.

2. a) Themistou E, Kanari A, Patrickios CS. J. Polym. Sci. Part A: Polym. Chem 2007;45:5811-5823. b) Skey J, O’Reilly RK. J. Polym. Sci. Part A: Polym. Chem 2008;46:3690-3702. c) Goh YK, Whittaker AK, Monteiro MJ. J. Polym. Sci. Part A: Polym. Chem 2007;45:4150-4153.

3. Moad G, Rizzardo E, Thang SH. Polymer 2008;49:1079-1131.

4. Matyjaszewski K, Xia J. Chem. Rev 2001;101:2921-2990. [PubMed: 11749397]

5. Hawker CJ, Bosman AW, Harth E. Chem. Rev 2001;101:3661-3688. [PubMed: 11740918]

6. a) Hawker CJ, Wooley KL. Science 2005;309:1200-1205. [PubMed: 16109874] b) Kloxin AM, Kasko AM, Salinas CN, Anseth KS. Science 2009;324:59-63. [PubMed: 19342581]

7. Oh JK, Siegwart DJ, Lee HI, Sherwood G, Peteanu L, Hollinger JO, Kataoka K, Matyjaszewski K. J. Am. Chem. Soc 2007;129:5939-5945. [PubMed: 17439215]

8. Paramonov SE, Bachelder EM, Beaudette TT, Standley SM, Lee CC, Dashe J, Fréchet JMJ. Bioconjug. Chem 2008;19:911-919. [PubMed: 18373356]

9. Cohen JL, Almutairi A, Cohen JA, Bernstein M, Brody SL, Schuster DP, Fréchet JMJ. Bioconjug. Chem 2008;19:876-881. [PubMed: 18318462]

10. Themistou E, Patrickios CS. Macromolecules 2004;37:6734-6743.

11. Gao H, Ohno S, Matyjaszewski K. J. Am. Chem. Soc 2006;128:15111-15113. [PubMed: 17117861]

12. Tsarevsky NV, Matyjaszewski K. Macromolecules 2005;38:3087-3092.

13. Nicolay R, Marx L, Hemery P, Matyjaszewski K. Macromolecules 2007;40:9217-9223.

14. Barner-Kowollik C, Perrier SJ. Polym. Sci. PartA: Polym. Chem 2008;46:5715-5723.

15. Xu J, He J, Fan D, Wang X, Yang Y. Macromolecules 2006;39:8616-8624.

16. a) You YZ, Manickam DS, Zhou QH, Oupický D. J. Control. Release 2007;122(3):217-225.

[PubMed: 17574292] b) Tao HE, Ying-Fang Z, Pan CY. Polym. J 2002;34:138-143. c) Whittaker MR, Goh Y-K, Gemici H, Legge TM, Perrier S, Monteiro MJ. Macromolecules 2006;39:90289034.Gemici H, Legge TM, Whittaker M, Monteiro MJ, Perrier S. J. Polym. Sci., Part A: Polym. Chem 2007;45:2334-2340.

17. Sanda F, Endo T. J. Polym. Sci. Part A: Polym. Chem 2001;39:265-276.

18. Bailey WJ, Wu S, Ni Z. Makromol. Chem 1982;183:1913-1920. 
19. Huang J, Gil R, Matyjaszewski K. Polymer 2005;46:11698-11706.

20. Evans RA, Moad G, Rizzardo E, Thang SH. Macromolecules 1994;27:7935-7937.

21. Rizzardo E, Evans RA, Moad G, Thang SH. PCT Int. Pat. Appl. 1994 WO 9414792.

22. Evans RA, Rizzardo E. Macromolecules 1996;29:6983-6989.

23. Chaumont P, Asgarzadeh F, Colombani D, Arotcarena M, Baudouin A. Macromol. Chem. Phys 1998;199:2577-2582.

24. Phelan M, Aldabbagh F, Zetterlund PB, Yamada B. Polymer 2005;46:12046-12056.

25. Meijs GF, Morton TC, Rizzardo E, Thang SH. Macromolecules 1991;24:3689-3695.

26. Mukaiyama T. Angew. Chem. Int. Ed. Engl 1979;18:707-721.

27. Perrier S, Takolpuckdee P, Westwood J, Lewis DM. Macromolecules 2004;37:2709-2717.

28. Arkles B, King K, Anderson R, Peterson W. Organometallics 1983;2:454-457.

29. Wallace OB, Springer DM. Tetrahedron Lett 1998;39:2693-2694.

30. Maiti S, Spevak P, Singh M, Micetich R, Reddy A. Synth. Commun 1988;18:575-581.

31. Khan SA, Erickson BW. J. Am. Chem. Soc 1981;103:7374-7376.

32. Weaver JVM, Bannister I, Robinson KL, Bories-Azeau X, Armes SP, Smallridge M, McKenna P. Macromolecules 2004;37:2395-2403. 


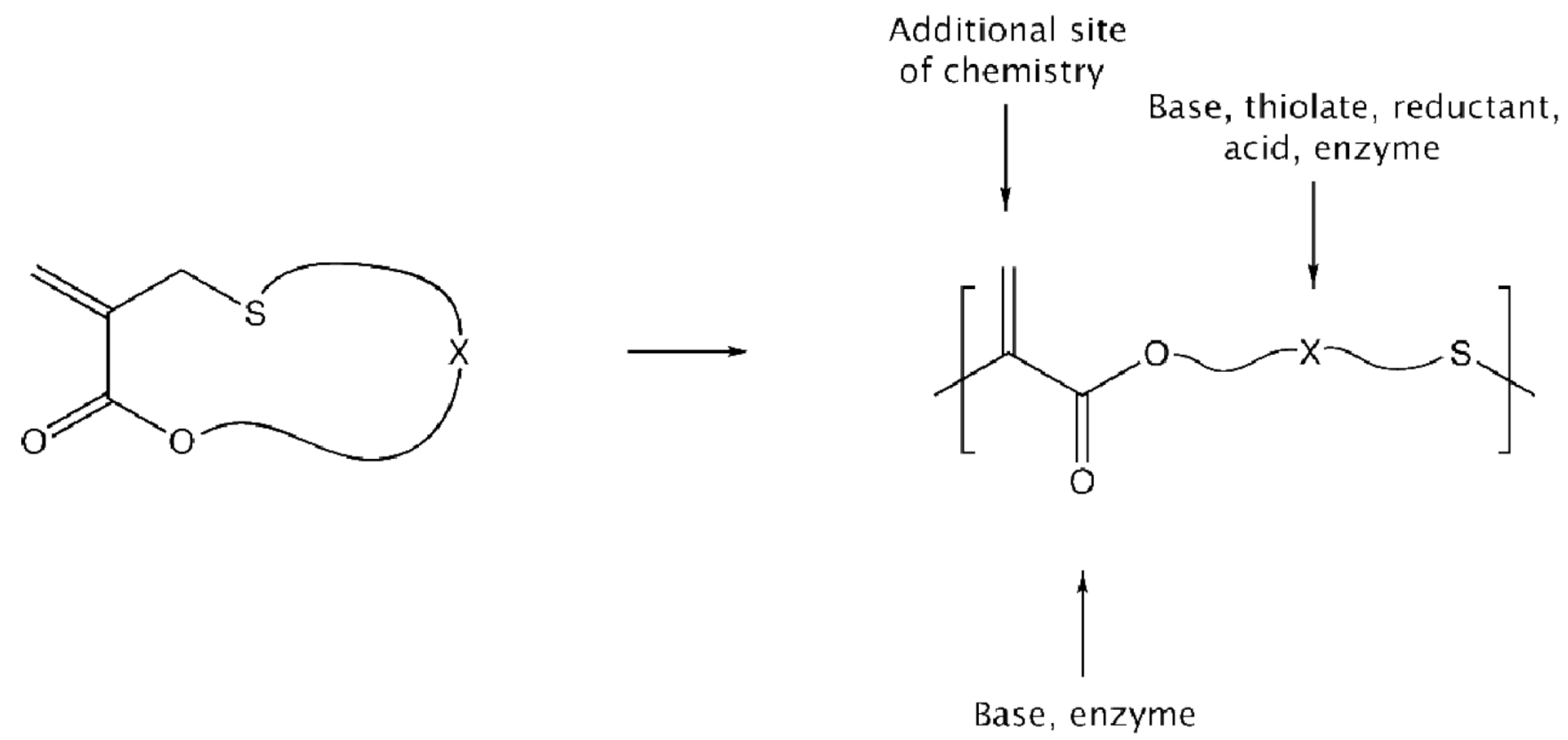

Figure 1.

Schematic representation of the building block nature of the cyclic monomers and their ability to introduce, multiple, disparate functional groups into the polymer chain. 

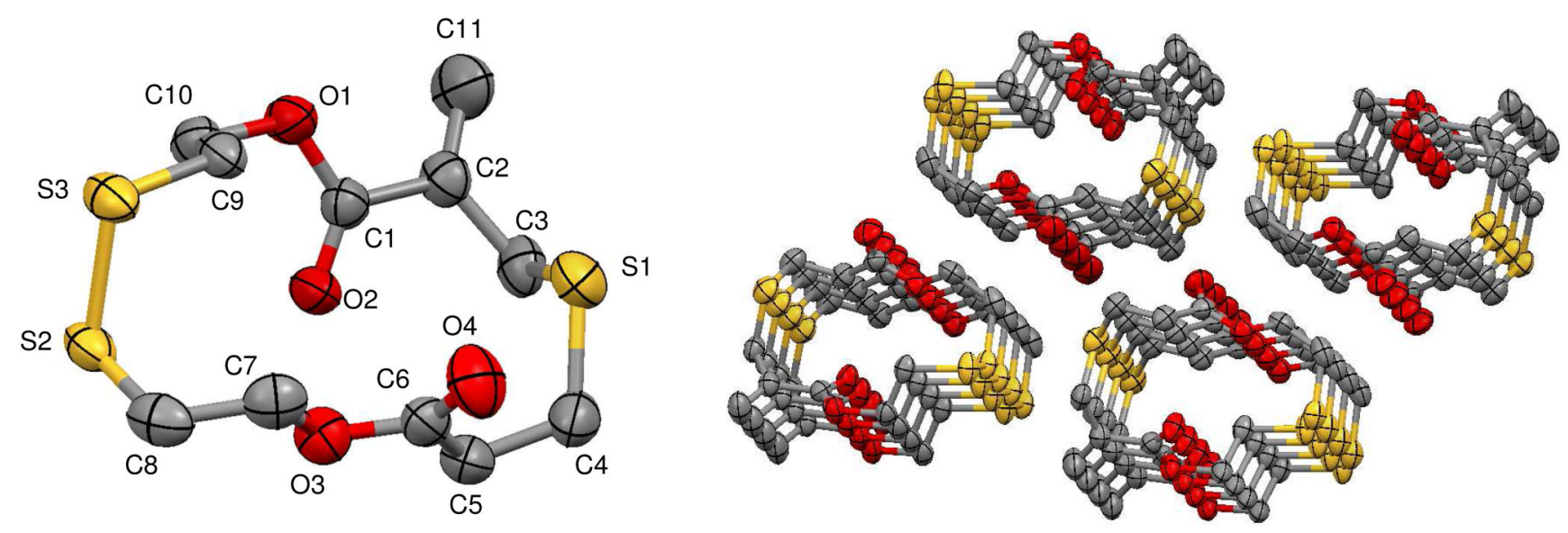

Figure 2.

Molecular structure and crystal packing of monomer 4 . Thermal ellipsoids are drawn at the $50 \%$ probability level, and hydrogen atoms have been omitted for clarity. 

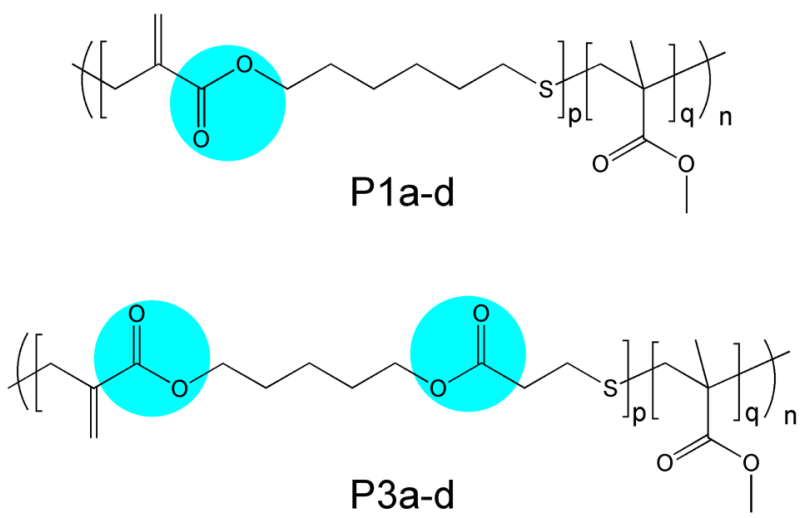

basic degradation
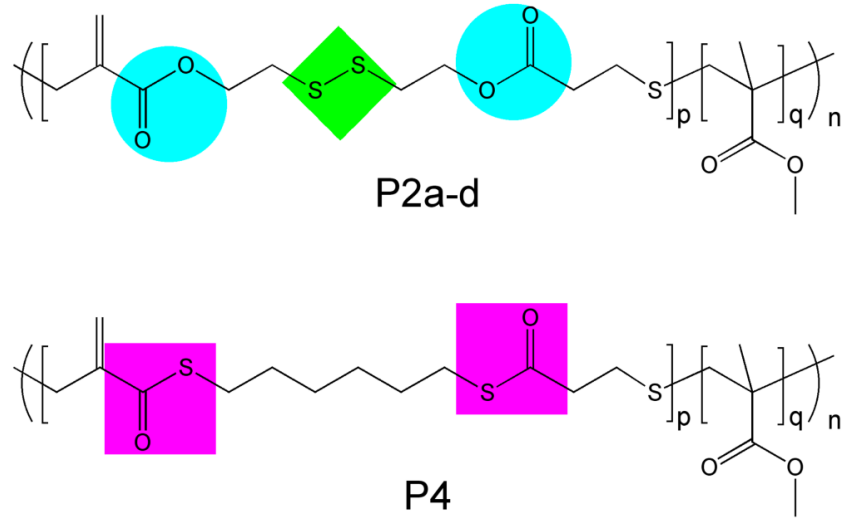

thiolate degradation

Figure 3.

Copolymers P1-4 based on MMA and cyclic monomers 2, 4, 5 and 6 respectively and degradation conditions for specific cyclic allylic monomers. 

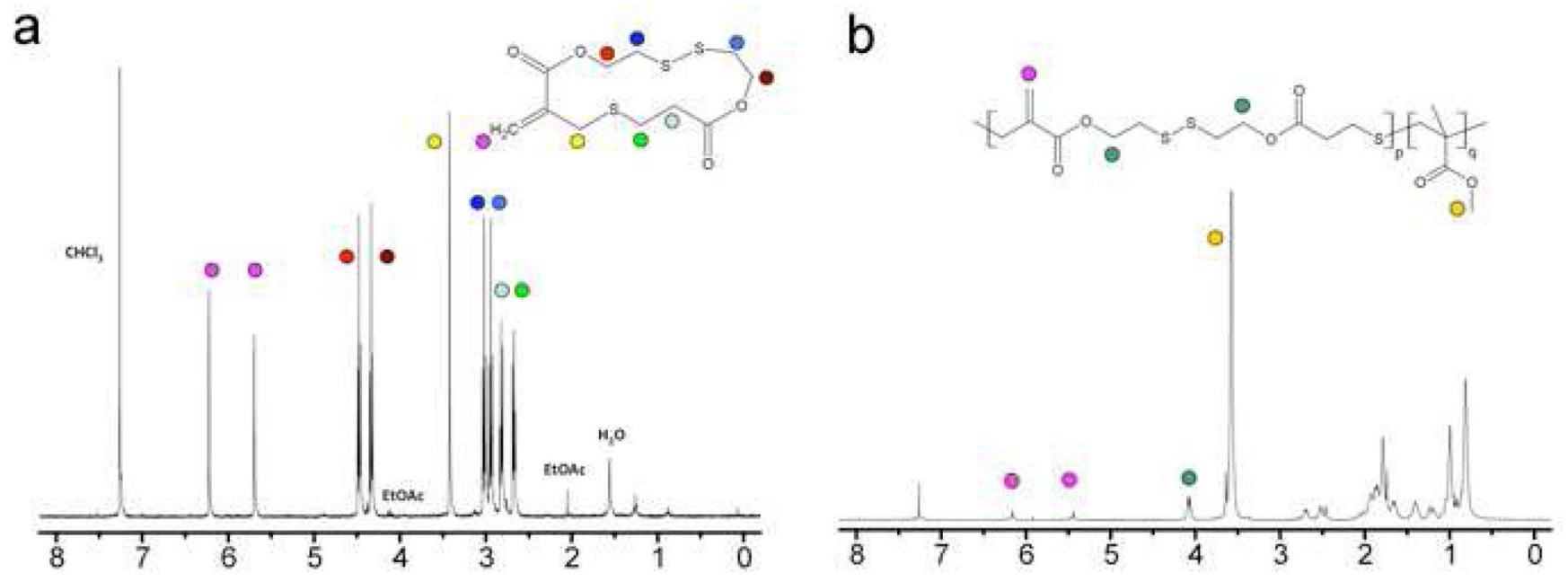

Figure 4.

${ }^{1} \mathrm{H}$ NMR for the disulphide monomer, $\mathbf{4}$, and the corresponding copolymer with MMA, P2. 

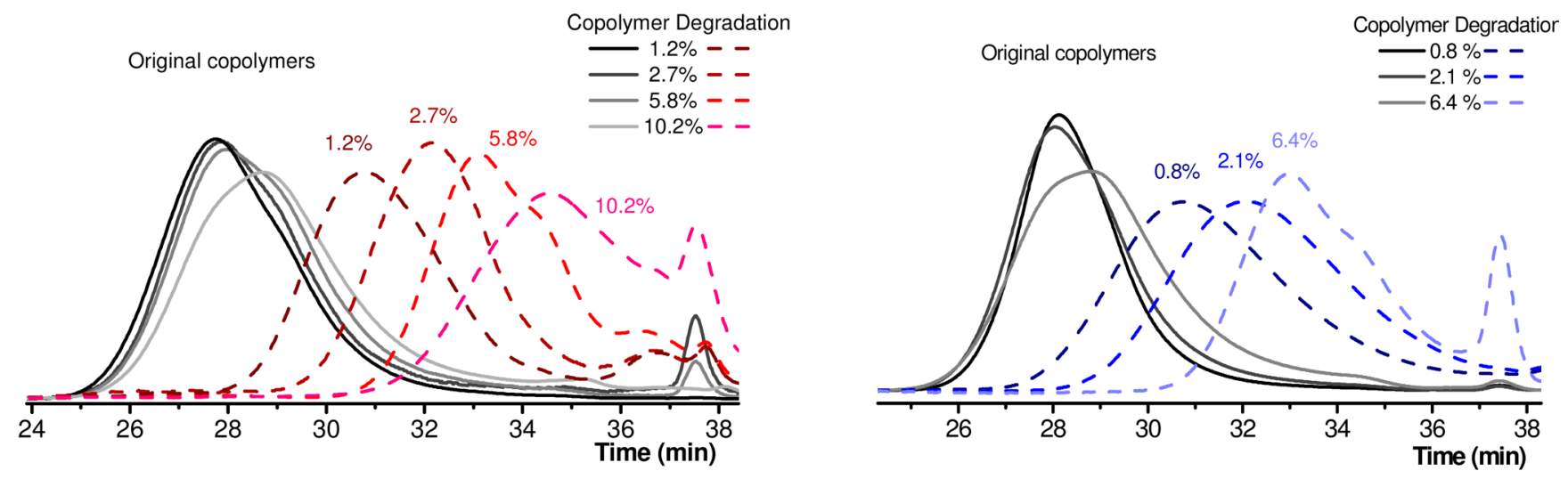

Figure 5.

SEC traces of copolymers P1a-d (left) derived from MMA/2 and P2a-c (right) derived from MMA/4 and their corresponding degradation products (dashed lines). Percentage values indicate molar \% of cyclic allylic sulfide monomer in polymer. 


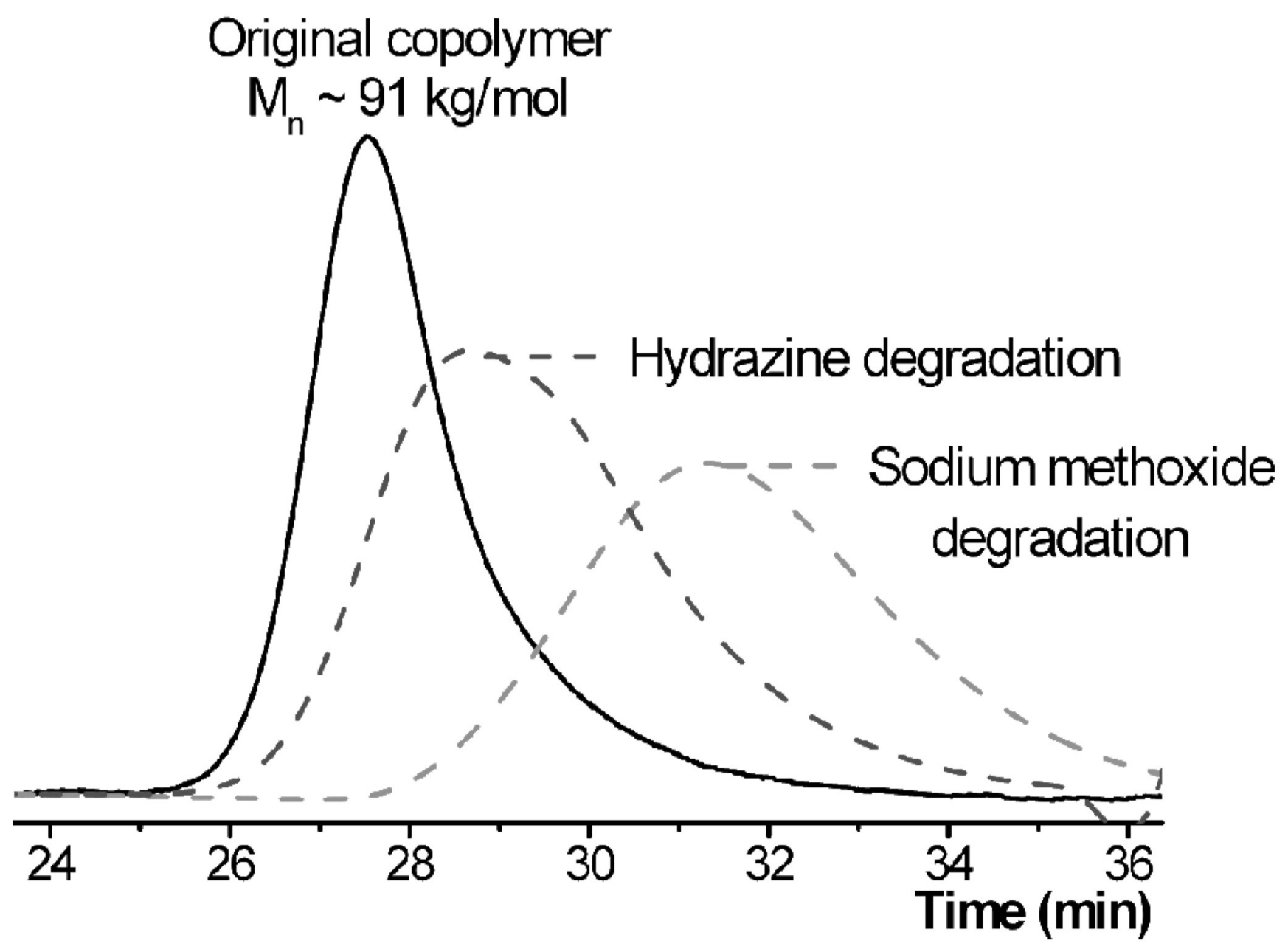

Figure 6.

SEC traces of mixed copolymer P15 and its products after step-wise degradation. 

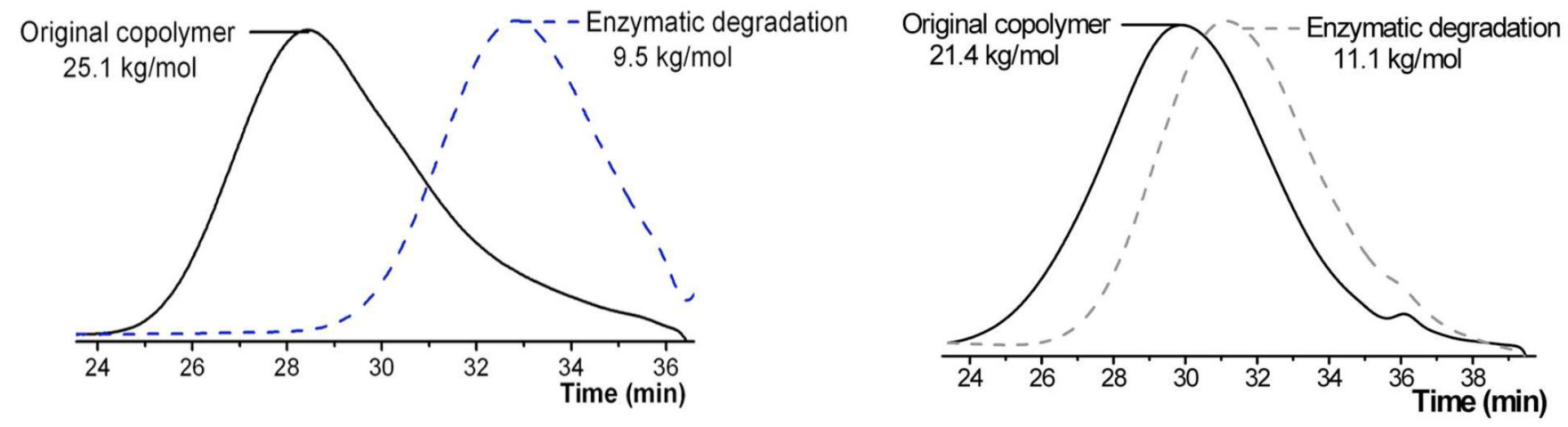

Figure 7.

SEC traces of copolymer P7 and P9, and their products after enzymatic degradation. 


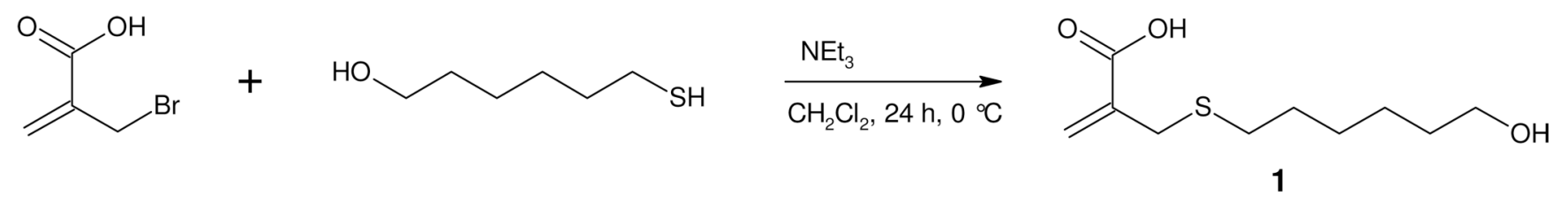<smiles>C[n+]1ccccc1Cl</smiles><smiles>C=C(CSC)CSCCCCCCOC(=O)C(=C)C</smiles>

Scheme 1.

Synthesis of cyclic monomer $\mathbf{2}$ via cyclization of the hydroxyl acid $\mathbf{1}$ under high dilution conditions. 
<smiles>C=C(CSCCC(=O)OC(=O)C(=C)C(=O)OC(=O)C(=C)CBr)C(=O)O</smiles><smiles>C=C(CSCCC(=O)Cl)C(=O)Cl</smiles>

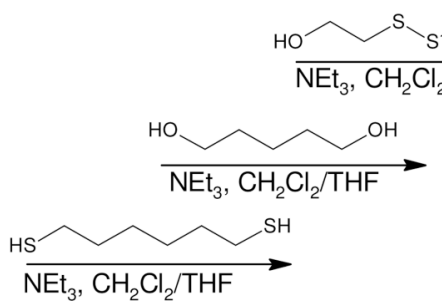<smiles>C=C(CSC)C(=O)SCC</smiles>

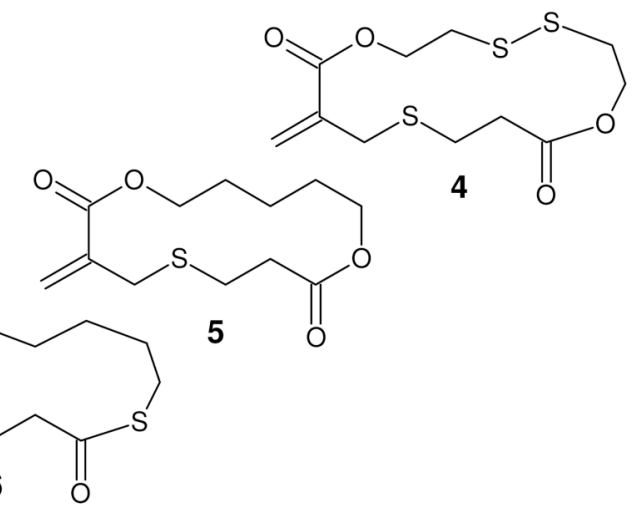

Scheme 2.

Synthesis of cyclic monomers 4-6 via the acid chloride route. 
<smiles>C=C(CSCCC(=O)Cl)C(=O)Cl</smiles>
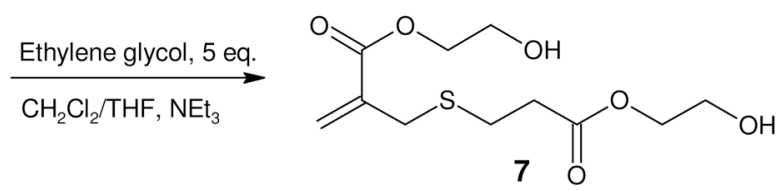
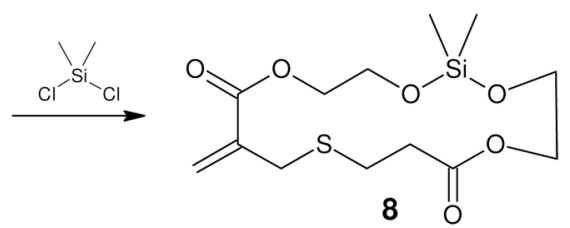

Scheme 3.

Synthesis of silyl-ether monomer $\mathbf{8}$ from bis-alcohol 7. 


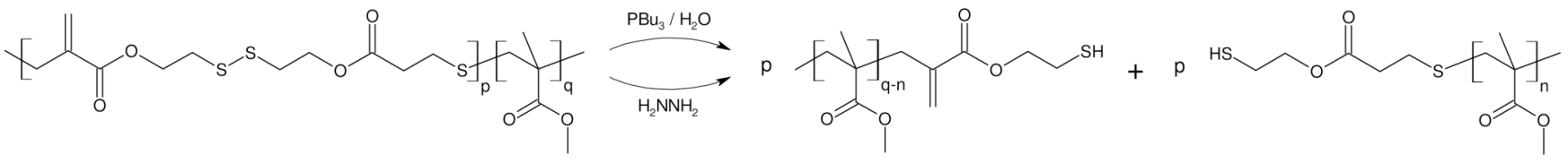

Scheme 4.

Cleavage of disulfides in copolymers P2a-c. 